\title{
Frame sequences and dual frames for operators
}

\author{
Zhong-Qi Xiang*, Yong-Ming Li \\ College of Mathematics and Computer Science, Shangrao Normal University, 401 Zhimin Street, \\ Xinzhou District, Shangrao 334001, Jiangxi Province, China
}

*Corresponding author, e-mail: lxsy20110927@163.com

Received 27 Oct 2015

Accepted 7 Jun 2016

\begin{abstract}
We introduce the more general frame sequences and dual frames related to a linear bounded operator $K$ in Hilbert spaces which we call $K$-frame sequences and dual $K$-frames, respectively. We give several equivalent characterizations for $K$-frame sequences. We also investigate the relationships among $K$-frame sequences, $K$-frames, and frame sequences, and give a new perturbation result for $K$-frames by using the associated dual $K$-frames. It turns out that in many ways $K$-frame sequences and dual $K$-frames behave completely differently from frame sequences and dual frames, respectively.
\end{abstract}

KEYWORDS: $K$-frame, $K$-frame sequence, dual $K$-frame, perturbation

MSC2010: 42C15 42C40

\section{INTRODUCTION}

Let $\mathscr{H}$ be a separable Hilbert space over the complex field. A sequence $\left\{f_{j}\right\}_{j=1}^{\infty}$ in $\mathscr{H}$ is a frame (an ordinary frame) if there exist constants $0<A \leqslant B<$ $\infty$ such that

$$
A\|f\|^{2} \leqslant \sum_{j=1}^{\infty}\left|\left\langle f, f_{j}\right\rangle\right|^{2} \leqslant B\|f\|^{2}, \quad \forall f \in \mathscr{H} .
$$

The sequence $\left\{f_{j}\right\}_{j=1}^{\infty}$ is said to be a Bessel sequence for $\mathscr{H}$ if we only require the right-hand inequality of (1). If (1) holds only for each $f \in \overline{\operatorname{span}}\left\{f_{j}\right\}_{j=1}^{\infty}$, then we call $\left\{f_{j}\right\}_{j=1}^{\infty}$ a frame sequence, where $\overline{\operatorname{span}} S$ denotes the closed linear span of sequence $S$.

One of the essential applications of frames is that they provide basis-like but generally nonunique decompositions for the elements of $\mathscr{H}$. In these decompositions, dual frames play a key role. Recall that a Bessel sequence $\left\{g_{j}\right\}_{j=1}^{\infty}$ in $\mathscr{H}$ is called a dual frame for the frame $\left\{f_{j}\right\}_{j=1}^{\infty}$ if

$$
f=\sum_{j=1}^{\infty}\left\langle f, g_{j}\right\rangle f_{j}, \quad \forall f \in \mathscr{H} .
$$

Owing to the redundancy and flexibility, frames have applications such as in wireless communication $^{1}, \Sigma \Delta$ quantization $^{2}$, sampling theory ${ }^{3}$, and image processing ${ }^{4}$. For details and background on frames see Refs. 5-8.

Găvruţa ${ }^{9}$ recently presented a generalization of ordinary frames with a linear bounded operator $K$, named $K$-frames, when working on atomic systems for operators. From Ref. 9 we know that $K$-frames possess higher generality than ordinary frames in the sense that the lower frame bound condition holds only for the elements in the range of $K$ and that they allow the reconstruction of the elements from the range of $K$ in a stable way and, in general, the range is not even a closed space. Hence $K$ frames provide more flexibility and thus make the study of them interesting. Note also that there are many essential differences between $K$-frames and ordinary frames due to the involved operator $K$. For instance, we know that an important equivalent characterization of ordinary frames is that the corresponding synthesis operators are bounded and surjective. But for $K$-frames, it is required that the corresponding synthesis operators are bounded and the range of $K$ is included in the ranges of the synthesis operators (see Theorem 4 in Ref. 9). Moreover, the roles of the dual $K$-frame pair cannot be interchanged in general (see Example 3.2 in Ref. 10), and a $K$-frame does not admit a dual frame in general (see Example 4 in this paper). For more details on $K$-frames, see Refs. 11-13.

In the study of $K$-frame theory, we often need to consider sequences which cannot form $K$-frames for the whole space or we are only interested in expansions for subspaces in some cases. Motivated by this and the fact that the properties of $K$-frames are quite different from those of ordinary frames, we apply Găvruţa's idea in the present paper to 
introduce the so-called $K$-frame sequences and investigate their properties. As mentioned above, $K$ frames are a generalization of ordinary frames and the dual frame is a very useful concept in frame theory. Thus it is natural to extend the dual for frames to the case of $K$-frames and examine its properties.

The paper is organized in the following manner. We continue this introductory section with a review of some basic definitions and facts on $K$-frames and operators. In the next section we study the equivalent characterization of $K$-frame sequences and the relationships among $K$-frame sequences, $K$-frames, and frame sequences. The third section deals with the stability of $K$-frames under perturbations.

Let $\mathscr{H}_{1}$ and $\mathscr{H}_{2}$ be two Hilbert spaces. We denote by $L\left(\mathscr{H}_{1}, \mathscr{H}_{2}\right)$ the set of all linear bounded operators from $\mathscr{H}_{1}$ to $\mathscr{H}_{2}$ and $L\left(\mathscr{H}_{1}, \mathscr{H}_{1}\right)$ is abbreviated by $L\left(\mathscr{H}_{1}\right)$. For $\Lambda \in L\left(\mathscr{H}_{1}, \mathscr{H}_{2}\right)$, we use $R(\Lambda)$ to denote the range of $\Lambda$. Suppose that the operator $Q \in L\left(\mathscr{H}_{1}, \mathscr{H}_{2}\right)$ has a closed range. Then there exists a unique operator $Q^{\dagger} \in L\left(\mathscr{H}_{2}, \mathscr{H}_{1}\right)$, called the pseudo-inverse of $Q$, satisfying

$$
\begin{gathered}
Q Q^{\dagger} Q=Q, \quad Q^{\dagger} Q Q^{\dagger}=Q^{\dagger}, \\
\left(Q Q^{\dagger}\right)^{*}=Q Q^{\dagger}, \quad\left(Q^{\dagger} Q\right)^{*}=Q^{\dagger} Q
\end{gathered}
$$

In the following we always assume that the operator $K \in L(\mathscr{H})$ is not equal to zero and that it has a closed range. We use $\Theta^{\dagger}$ to denote the pseudo-inverse of the linear bounded operator $\Theta$ (if it exists).

Definition 1 [Ref. 9] A sequence $\left\{f_{j}\right\}_{j=1}^{\infty} \subset \mathscr{H}$ is called a $K$-frame for $\mathscr{H}$ if there exist two constants $0<C \leqslant D<\infty$ such that

$$
\begin{aligned}
C\left\|K^{*} f\right\|^{2} & \leqslant \sum_{j=1}^{\infty}\left|\left\langle f, f_{j}\right\rangle\right|^{2} \\
& \leqslant D\|f\|^{2}, \quad \forall f \in \mathscr{H} .
\end{aligned}
$$

The numbers $C, D$ are called $K$-frame bounds. If the above inequalities hold only for each $f \in$ $\overline{\operatorname{span}}\left\{f_{j}\right\}_{j=1}^{\infty}$, then $\left\{f_{j}\right\}_{j=1}^{\infty}$ is said to be a $K$-frame sequence.

Remark 1 If $K$ is equal to $\operatorname{Id}_{\mathscr{H}}$, the identity operator on $\mathscr{H}$, then the $K$-frames and $K$-frame sequences are just ordinary frames and frame sequences, respectively.

Lemma 1 (Ref. 8) Let $\left\{f_{j}\right\}_{j=1}^{\infty}$ be a Bessel sequence for $\mathscr{H}$. Then $\sum_{j=1}^{\infty} c_{j} f_{j}$ converges unconditionally for each $\left\{c_{j}\right\}_{j=1}^{\infty} \in \ell^{2}(\mathbb{N})$ and the operators defined by

$$
\begin{array}{r}
T: \ell^{2}(\mathbb{N}) \rightarrow \mathscr{H}, \quad T\left\{c_{j}\right\}_{j=1}^{\infty}=\sum_{j=1}^{\infty} c_{j} f_{j} \\
\text { (synthesis operator) }
\end{array}
$$

$$
T^{*}: \mathscr{H} \rightarrow \ell^{2}(\mathbb{N}), \quad T^{*} f=\left\{\left\langle f, f_{j}\right\rangle\right\}_{j=1}^{\infty}
$$

(analysis operator)

$$
\begin{aligned}
& S: \mathscr{H} \rightarrow \mathscr{H}, \quad S f=T T^{*} f=\sum_{j=1}^{\infty}\left\langle f, f_{j}\right\rangle f_{j} \\
& \text { (frame operator) }
\end{aligned}
$$

are linear and bounded.

If $\left\{f_{j}\right\}_{j=1}^{\infty}$ is a Bessel sequence for $\overline{\operatorname{span}}\left\{f_{j}\right\}_{j=1}^{\infty}$, then, by replacing $\mathscr{H}$ in (3)-(5) with $\overline{\operatorname{span}}\left\{f_{j}\right\}_{j=1}^{\infty}$, we will still obtain the associated operators of $\left\{f_{j}\right\}_{j=1}^{\infty}$. Clearly, if $\left\{f_{j}\right\}_{j=1}^{\infty}$ is a frame sequence, then the corresponding synthesis operator $T$ and frame operator $S$ are, respectively, surjective and invertible. In this case, the following reconstruction formula is satisfied.

$$
\begin{aligned}
& f=\sum_{j=1}^{\infty}\left\langle f, f_{j}\right\rangle S^{-1} f_{j}=\sum_{j=1}^{\infty}\left\langle f, S^{-1} f_{j}\right\rangle f_{j}, \\
& \forall f \in \overline{\operatorname{span}}\left\{f_{j}\right\}_{j=1}^{\infty} .
\end{aligned}
$$

Later we will also need the following important result from operator theory.

Lemma 2 (Ref. 14) Let $U \in L\left(\mathscr{H}_{1}, \mathscr{H}\right)$ and $V \in$ $L\left(\mathscr{H}_{2}, \mathscr{H}\right)$. Then the following conditions are equivalent:

(i) $R(U) \subset R(V)$;

(ii) there exists $\lambda>0$ such that $U U^{*} \leqslant \lambda V V^{*}$;

(iii) there exists $\theta \in L\left(\mathscr{H}_{1}, \mathscr{H}_{2}\right)$ such that $U=V \theta$.

\section{$K$-FRAME SEQUENCES IN HILBERT SPACES}

In general, a $K$-frame for $\mathscr{H}$ is a $K$-frame sequence, but not conversely.

Example 1 Let $\left\{e_{j}\right\}_{j=1}^{\infty}$ be an orthonormal basis for $\mathscr{H}$. Fix $N \in \mathbb{N}$ and define $K \in L(\mathscr{H})$ as follows:

$$
K e_{j}= \begin{cases}j e_{j}, & 1 \leqslant j \leqslant N \\ e_{j}, & j>N .\end{cases}
$$


It is easily seen that $K^{*} e_{j}=K e_{j}$. For any $f \in$ $\overline{\operatorname{span}}\left\{e_{j}\right\}_{j=N+1}^{\infty}$, we have

$$
f=\sum_{j=N+1}^{\infty}\left\langle f, e_{j}\right\rangle e_{j} .
$$

Thus $K^{*} f=\sum_{j=N+1}^{\infty}\left\langle f, e_{j}\right\rangle e_{j}$. Hence

$$
\left\|K^{*} f\right\|^{2}=\sum_{j=N+1}^{\infty}\left|\left\langle f, e_{j}\right\rangle\right|^{2},
$$

which implies that $\left\{e_{j}\right\}_{j=N+1}^{\infty}$ is a $K$-frame for $\overline{\operatorname{span}}\left\{e_{j}\right\}_{j=N+1}^{\infty}$. If $\left\{e_{j}\right\}_{j=N+1}^{\infty}$ is a $K$-frame for $\mathscr{H}$ with bounds $C, D$, we let $e_{1} \in \mathscr{H}$. Then we have

$$
\sum_{j=N+1}^{\infty}\left|\left\langle e_{1}, e_{j}\right\rangle\right|^{2}=0 \geqslant C\left\|K^{*} e_{1}\right\|^{2}=C\left\|e_{1}\right\|^{2}=C .
$$

This is impossible. Hence $\left\{e_{j}\right\}_{j=N+1}^{\infty}$ is not a $K$-frame for $\mathscr{H}$.

We now give a condition under which a $K$-frame sequence is a $K$-frame.

Theorem 1 Let $\left\{f_{j}\right\}_{j=1}^{\infty}$ be a $K$-frame sequence in $\mathscr{H}$ with bounds $C$, $D$. If $R(K) \subset \overline{\operatorname{span}}\left\{f_{j}\right\}_{j=1}^{\infty}$, then $\left\{f_{j}\right\}_{j=1}^{\infty}$ is a $\mathrm{K}$-frame for $\mathscr{H}$.

Proof: We first show that $\left\{f_{j}\right\}_{j=1}^{\infty}$ is a Bessel sequence for $\mathscr{H}$. Since

$$
\mathscr{H}=\overline{\operatorname{span}}\left\{f_{j}\right\}_{j=1}^{\infty} \oplus\left(\overline{\operatorname{span}}\left\{f_{j}\right\}_{j=1}^{\infty}\right)^{\perp},
$$

for every $f \in \mathscr{H}$ there exist $g_{1} \in \overline{\operatorname{span}}\left\{f_{j}\right\}_{j=1}^{\infty}, g_{2} \in$ $\left(\overline{\operatorname{span}}\left\{f_{j}\right\}_{j=1}^{\infty}\right)^{\perp}$ such that $f=g_{1}+g_{2}$. Noting $\left\langle f, f_{j}\right\rangle=\left\langle g_{1}, f_{j}\right\rangle$ for each $j \in \mathbb{N}$, we obtain

$$
\begin{aligned}
\sum_{j=1}^{\infty}\left|\left\langle f, f_{j}\right\rangle\right|^{2} & =\sum_{j=1}^{\infty}\left|\left\langle g_{1}, f_{j}\right\rangle\right|^{2} \\
& \leqslant D\left\|g_{1}\right\|^{2} \leqslant D\left(\left\|g_{1}\right\|^{2}+\left\|g_{2}\right\|^{2}\right)=D\|f\|^{2} .
\end{aligned}
$$

We next prove the lower $K$-frame bound condition. As mentioned before, every $f \in \mathscr{H}$ has a decomposition as $f=g_{1}+g_{2}$, where $g_{1} \in \overline{\operatorname{span}}\left\{f_{j}\right\}_{j=1}^{\infty}$ and $g_{2} \in$ $\left(\overline{\operatorname{span}}\left\{f_{j}\right\}_{j=1}^{\infty}\right)^{\perp}$. Since $R(K) \subset \overline{\operatorname{span}}\left\{f_{j}\right\}_{j=1}^{\infty}$, it follows that $g_{2} \in(R(K))^{\perp}$. It is obvious that $\left\langle K^{*} g_{2}, h\right\rangle=$ $\left\langle g_{2}, K h\right\rangle=0$ for all $h \in \mathscr{H}$. Thus $K^{*} g_{2}=0$. Now

$$
\begin{aligned}
& \sum_{j=1}^{\infty}\left|\left\langle f, f_{j}\right\rangle\right|^{2}=\sum_{j=1}^{\infty}\left|\left\langle g_{1}, f_{j}\right\rangle\right|^{2} \\
& \quad \geqslant C\left\|K^{*} g_{1}\right\|^{2}=C\left\|K^{*}\left(g_{1}+g_{2}\right)\right\|^{2}=C\left\|K^{*} f\right\|^{2} .
\end{aligned}
$$

The following result shows that we can obtain a $K$-frame sequence from a frame sequence.

Theorem 2 Every frame sequence in $\mathscr{H}$ is a $\mathrm{K}$-frame sequence.

Proof: Suppose that $\left\{f_{j}\right\}_{j=1}^{\infty}$ is a frame sequence with bounds $C, D$ and the frame operator $S$. To prove that $\left\{f_{j}\right\}_{j=1}^{\infty}$ is a $K$-frame sequence, it is sufficient to prove, by Theorem 1 , that the lower $K$-frame bound condition holds. For each $f \in \overline{\operatorname{span}}\left\{f_{j}\right\}_{j=1}^{\infty}$, the reconstruction formula gives $f=\sum_{j=1}^{\infty}\left\langle f, f_{j}\right\rangle S^{-1} f_{j}$ and, consequently, $K^{*} f=\sum_{j=1}^{\infty}\left\langle f, f_{j}\right\rangle K^{*} S^{-1} f_{j}$. Denote by $P_{\overline{s p a n}}\left\{f_{j}\right\}_{j=1}^{\infty}$ the orthogonal projection on $\overline{\operatorname{span}}\left\{f_{j}\right\}_{j=1}^{\infty}$. Then

$$
\begin{gathered}
\left\|K^{*} f\right\|=\sup _{\|g\|=1}\left|\sum_{j=1}^{\infty}\left\langle f, f_{j}\right\rangle\left\langle K^{*} P_{\overline{\mathrm{span}}\left\{f_{j}\right\}_{j=1}^{\infty}} S^{-1} f_{j}, g\right\rangle\right| \\
\leqslant \sup _{\|g\|=1}\left(\sum_{j=1}^{\infty}\left|\left\langle S^{-1} P_{\overline{\mathrm{spa}}\left\{f_{j}\right\}_{j=1}^{\infty}} K g, f_{j}\right\rangle\right|^{2}\right)^{1 / 2} \\
\times\left(\sum_{j=1}^{\infty}\left|\left\langle f, f_{j}\right\rangle\right|^{2}\right)^{1 / 2} \\
\leqslant \sqrt{D}\left\|S^{-1} P_{\overline{\mathrm{span}}\left\{f_{j}\right\}_{j=1}^{\infty}} K\right\|\left(\sum_{j=1}^{\infty}\left|\left\langle f, f_{j}\right\rangle\right|^{2}\right)^{1 / 2} \\
\leqslant \sqrt{D}\left\|S^{-1}\right\|\|K\|\left(\sum_{j=1}^{\infty}\left|\left\langle f, f_{j}\right\rangle\right|^{2}\right)^{1 / 2} .
\end{gathered}
$$

It follows that

$$
D^{-1}\left\|S^{-1}\right\|^{-2}\|K\|^{-2}\left\|K^{*} f\right\|^{2} \leqslant \sum_{j=1}^{\infty}\left|\left\langle f, f_{j}\right\rangle\right|^{2},
$$

as desired.

One may wonder whether the converse of Theorem 2 holds. In fact, the answer is negative.

Example 2 Let $\left\{e_{j}\right\}_{j=1}^{\infty}$ be an orthonormal basis for $\mathscr{H}$ and define

$$
K: \mathscr{H} \rightarrow \mathscr{H}, \quad K f=\sum_{j=1}^{\infty}\left\langle f, e_{2 j}\right\rangle e_{2 j} .
$$

Clearly, $K$ is a well defined, linear bounded operator with $K^{*} f=\sum_{j=1}^{\infty}\left\langle f, e_{2 j}\right\rangle e_{2 j}$. Let

$$
f_{j}= \begin{cases}e_{j}, & j \text { is even, } \\ e_{j} / j, & j \text { is odd. }\end{cases}
$$


For any $f \in \overline{\operatorname{span}}\left\{f_{j}\right\}_{j=1}^{\infty}$ we have

$$
\begin{aligned}
\left\|K^{*} f\right\|^{2} & =\sum_{j=1}^{\infty}\left|\left\langle f, e_{2 j}\right\rangle\right|^{2} \leqslant \sum_{j=1}^{\infty}\left|\left\langle f, f_{j}\right\rangle\right|^{2} \\
& =\sum_{j=1}^{\infty}\left|\left\langle f, e_{2 j}\right\rangle\right|^{2}+\sum_{j=1}^{\infty} \frac{\left|\left\langle f, e_{2 j-1}\right\rangle\right|^{2}}{(2 j-1)^{2}} \\
& \leqslant \sum_{j=1}^{\infty}\left|\left\langle f, e_{j}\right\rangle\right|^{2}=\|f\|^{2} .
\end{aligned}
$$

Thus $\left\{f_{j}\right\}_{j=1}^{\infty}$ is a $K$-frame sequence. We next prove that $\left\{f_{j}\right\}_{j=1}^{\infty}$ is not a frame sequence. Assume on the contrary that there is a constant $C>0$ such that $C\|f\|^{2} \leqslant \sum_{j=1}^{\infty}\left|\left\langle f, f_{j}\right\rangle\right|^{2}$ for all $f \in \overline{\operatorname{span}}\left\{f_{j}\right\}_{j=1}^{\infty}$. Let $k \in \mathbb{N}$ be a positive integer which is greater than $1 / 2 \sqrt{C}+\frac{1}{2}$. Taking $e_{2 k-1} \in \overline{\operatorname{span}}\left\{f_{j}\right\}_{j=1}^{\infty}$, we obtain

$$
\begin{aligned}
C & =C\left\|e_{2 k-1}\right\|^{2} \leqslant \sum_{j=1}^{\infty}\left|\left\langle e_{2 k-1}, f_{j}\right\rangle\right|^{2} \\
& =\sum_{j=1}^{\infty}\left|\left\langle e_{2 k-1}, \frac{e_{2 j-1}}{2 j-1}\right\rangle\right|^{2}=\frac{1}{(2 k-1)^{2}}<C,
\end{aligned}
$$

which is a contradiction. Hence $\left\{f_{j}\right\}_{j=1}^{\infty}$ is not a frame sequence.

We are now ready to present the result showing that the converse of Theorem 1 remains true if we replace "frame sequence" by " $K$-frame sequence".

Theorem 3 Let $\left\{f_{j}\right\}_{j=1}^{\infty}$ be a frame sequence in $\mathscr{H}$ with synthesis operator $T$. Then it is a $K$-frame for $\mathscr{H}$ if and only if $R(K) \subset \overline{\operatorname{span}}\left\{f_{j}\right\}_{j=1}^{\infty}$.

Proof: Assume first that $R(K) \subset \overline{\operatorname{span}}\left\{f_{j}\right\}_{j=1}^{\infty}$. By Theorem 2 we know that $\left\{f_{j}\right\}_{j=1}^{\infty}$ is a $K$-frame sequence in $\mathscr{H}$. From Theorem 1 it follows that $\left\{f_{j}\right\}_{j=1}^{\infty}$ is a $K$-frame for $\mathscr{H}$. Conversely, let us denote the $K$-frame bounds of $\left\{f_{j}\right\}_{j=1}^{\infty}$ by $C, D$. Then

$$
\begin{aligned}
\left\langle C K K^{*} f, f\right\rangle \leqslant & \sum_{j=1}^{\infty}\left|\left\langle f, f_{j}\right\rangle\right|^{2}=\left\|T^{*} P_{\overline{\operatorname{span}}\left\{f_{j}\right\}_{j=1}^{\infty}} f\right\|^{2} \\
& =\left\langle\left(T^{*} P_{\overline{\operatorname{span}}\left\{f_{j}\right\}_{j=1}^{\infty}}\right)^{*}\left(T^{*} P_{\overline{\operatorname{span}}\left\{f_{j}\right\}_{j=1}^{\infty}}\right) f, f\right\rangle
\end{aligned}
$$

for any $f \in \mathscr{H}$, that is,

$$
C K K^{*} \leqslant\left(T^{*} P_{\overline{\operatorname{span}}\left\{f_{j}\right\}_{j=1}^{\infty}}\right)^{*}\left(T^{*} P_{\overline{\operatorname{span}}\left\{f_{j}\right\}_{j=1}^{\infty}}\right) .
$$

Using Lemma 2 and the fact that $R(T)=$ $\overline{\operatorname{span}}\left\{f_{j}\right\}_{j=1}^{\infty}$, we obtain

$$
\begin{aligned}
& R(K) \subset R\left(\left(T^{*} P_{\overline{\operatorname{span}}\left\{f_{j}\right\}_{j=1}^{\infty}}\right)^{*}\right) \\
& =R\left(P_{\overline{\operatorname{span}}\left\{f_{j}\right\}_{j=1}^{\infty}} T\right)=\overline{\operatorname{span}}\left\{f_{j}\right\}_{j=1}^{\infty} .
\end{aligned}
$$

Although a $K$-frame sequence $\left\{f_{j}\right\}_{j=1}^{\infty}$ in $\mathscr{H}$ is not a frame for $\overline{\operatorname{span}}\left\{f_{j}\right\}_{j=1}^{\infty}$ in general, we show that it can be a frame for a closed subspace of $\overline{\operatorname{span}}\left\{f_{j}\right\}_{j=1}^{\infty}$.

Theorem 4 Let $\left\{f_{j}\right\}_{j=1}^{\infty}$ be a $K$-frame sequence in $\mathscr{H}$ with bounds $C, D$. Suppose that $\left.K^{*}\right|_{\overline{\operatorname{span}}\left\{f_{j}\right\}_{j=1}^{\infty}} \neq 0$ and that it has a closed range. Then $\left\{f_{j}\right\}_{j=1}^{\infty}$ is a frame for

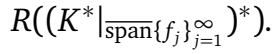

Proof: We conclude first that $\left(\left.K^{*}\right|_{\overline{\operatorname{span}}\left\{f_{j}\right\}_{j=1}^{\infty}}\right)^{*}$ has a closed range since, by assumption, $\left.K^{*}\right|_{\overline{\operatorname{span}}\left\{f_{j}\right\}_{j=1}^{\infty}}$ has a closed range. Hence the pseudo-inverse

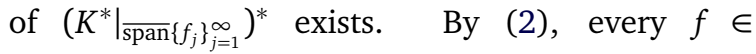

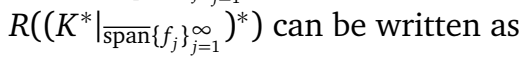

$$
\begin{aligned}
f & =\left(\left.K^{*}\right|_{\overline{\operatorname{span}}\left\{f_{j}\right\}_{j=1}^{\infty}}\right)^{*}\left(\left(\left.K^{*}\right|_{\overline{\operatorname{span}}\left\{f_{j}\right\}_{j=1}^{\infty}}\right)^{*}\right)^{\dagger} f \\
& =\left[\left(\left.K^{*}\right|_{\overline{\operatorname{span}}\left\{f_{j}\right\}_{j=1}^{\infty}}\right)^{*}\left(\left(\left.K^{*}\right|_{\overline{\operatorname{span}}\left\{f_{j}\right\}_{j=1}^{\infty}}\right)^{*}\right)^{\dagger}\right]^{*} f \\
& =\left(\left.K^{*}\right|_{\overline{\operatorname{span}}\left\{f_{j}\right\}_{j=1}^{\infty}}\right)^{\dagger}\left(\left.K^{*}\right|_{\overline{\operatorname{span}}\left\{f_{j}\right\}_{j=1}^{\infty}}\right) f .
\end{aligned}
$$

Hence

$$
\|f\|^{2} \leqslant\left\|\left(\left.K^{*}\right|_{\overline{\operatorname{span}}\left\{f_{j}\right\}_{j=1}^{\infty}}\right)^{\dagger}\right\|^{2}\left\|\left(\left.K^{*}\right|_{\overline{\operatorname{span}}\left\{f_{j}\right\}_{j=1}^{\infty}}\right) f\right\|^{2} .
$$

Notice, however, that $R\left(\left(\left.K^{*}\right|_{\overline{\operatorname{span}}\left\{f_{j}\right\}_{j=1}^{\infty}}\right)^{*}\right) \subseteq$ $\overline{\operatorname{span}}\left\{f_{j}\right\}_{j=1}^{\infty}$. We have

$$
\begin{aligned}
& \|f\|^{2} \leqslant\left\|\left(\left.K^{*}\right|_{\overline{\operatorname{span}}\left\{f_{j}\right\}_{j=1}^{\infty}}\right)^{\dagger}\right\|^{2}\left\|K^{*} f\right\|^{2}
\end{aligned}
$$

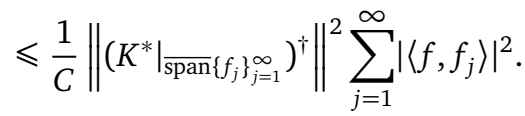

Since $\left.K^{*}\right|_{\overline{\operatorname{span}}\left\{f_{j}\right\}_{j=1}^{\infty}} \neq 0$, its pseudo-inverse $\left(\left.K^{*}\right|_{\overline{\operatorname{span}}\left\{f_{j}\right\}_{j=1}^{\infty}}\right)^{\dagger} \neq 0$. It follows that

$$
C\left\|\left(\left.K^{*}\right|_{\overline{\operatorname{span}}\left\{f_{j}\right\}_{j=1}^{\infty}}\right)^{\dagger}\right\|^{-2}\|f\|^{2} \leqslant \sum_{j=1}^{\infty}\left|\left\langle f, f_{j}\right\rangle\right|^{2} .
$$

It is trivial to show that

$$
\sum_{j=1}^{\infty}\left|\left\langle f, f_{j}\right\rangle\right|^{2} \leqslant D\|f\|^{2} \quad \forall f \in R\left(\left(\left.K^{*}\right|_{\overline{\operatorname{span}}\left\{f_{j}\right\}_{j=1}^{\infty}}\right)^{*}\right) .
$$

Thus $\left\{f_{j}\right\}_{j=1}^{\infty}$ is a frame for $R\left(\left(\left.K^{*}\right|_{\overline{\operatorname{span}}\left\{f_{j}\right\}_{j=1}^{\infty}}\right)^{*}\right)$ with bounds $C\left\|\left(\left.K^{*}\right|_{\overline{\operatorname{span}}\left\{f_{j}\right\}_{j=1}^{\infty}}\right)^{\dagger}\right\|^{-2}, D$.

At the end of this section we give several characterizations for $K$-frame sequences.

Theorem 5 Suppose that $\left\{f_{j}\right\}_{j=1}^{\infty}$ is a sequence in $\mathscr{H}$. Then the following statements are equivalent: 
(i) $\left\{f_{j}\right\}_{j=1}^{\infty}$ is a $K$-frame sequence;

(ii) $\left\{f_{j}\right\}_{j=1}^{\infty}$ is a Bessel sequence for $\overline{\operatorname{span}}\left\{f_{j}\right\}_{j=1}^{\infty}$ and there exits a Bessel sequence $\left\{g_{j}\right\}_{j=1}^{\infty}$ for $\mathscr{H}$ such that for any $f \in \mathscr{H}$,

$$
\left(\left.K^{*}\right|_{\overline{\operatorname{span}}\left\{f_{j}\right\}_{j=1}^{\infty}}\right)^{*} f=\sum_{j=1}^{\infty}\left\langle f, g_{j}\right\rangle f_{j} ;
$$

(iii) $\left\{f_{j}\right\}_{j=1}^{\infty}$ is a Bessel sequence for $\overline{\operatorname{span}}\left\{f_{j}\right\}_{j=1}^{\infty}$ and there exits a Bessel sequence $\left\{g_{j}\right\}_{j=1}^{\infty}$ for $\mathscr{H}$ such that for any $h \in \overline{\operatorname{span}}\left\{f_{j}\right\}_{j=1}^{\infty}$,

$$
K^{*} h=\sum_{j=1}^{\infty}\left\langle h, f_{j}\right\rangle g_{j} .
$$

Proof: (i) $\Rightarrow$ (ii). Let $C, D$ and $T$ be, respectively, the bounds and synthesis operator of $\left\{f_{j}\right\}_{j=1}^{\infty}$. Then for all $f \in \overline{\operatorname{span}}\left\{f_{j}\right\}_{j=1}^{\infty}$ we have $C\left\|K^{*} f\right\|^{2} \leqslant\left\|T^{*} f\right\|^{2}$, implying that

$$
C\left(\left.K^{*}\right|_{\overline{\operatorname{span}}\left\{f_{j}\right\}_{j=1}^{\infty}}\right)^{*}\left(\left.K^{*}\right|_{\overline{\operatorname{span}}\left\{f_{j}\right\}_{j=1}^{\infty}}\right) \leqslant T T^{*} .
$$

By Lemma 2, there exists $U \in L\left(\mathscr{H}, \ell^{2}(\mathbb{N})\right)$ such that $\left(\left.K^{*}\right|_{\overline{s p a n}\left\{f_{j}\right\}_{j=1}^{\infty}}\right)^{*}=T U$. Let $\left\{\delta_{j}\right\}_{j=1}^{\infty}$ be the canonical orthonormal basis for $\ell^{2}(\mathbb{N})$. Since

$$
\begin{aligned}
\left\langle f, f_{j}\right\rangle & =\left\langle\left\{\left\langle f, f_{j}\right\rangle\right\}_{j=1}^{\infty}, \delta_{j}\right\rangle=\left\langle T^{*} f, \delta_{j}\right\rangle \\
& =\left\langle f, T \delta_{j}\right\rangle, \quad \forall f \in \overline{\operatorname{span}}\left\{f_{j}\right\}_{j=1}^{\infty},
\end{aligned}
$$

we have $T \delta_{j}=f_{j}$ for all $j \in \mathbb{N}$. Taking $g_{j}=U^{*} \delta_{j}$ for each $j \in \mathbb{N}$,

$$
\begin{aligned}
\sum_{j=1}^{\infty}\left|\left\langle f, g_{j}\right\rangle\right|^{2} & =\sum_{j=1}^{\infty}\left|\left\langle U f, \delta_{j}\right\rangle\right|^{2}=\|U f\|^{2} \\
& \leqslant\|U\|^{2}\|f\|^{2}, \quad \forall f \in \mathscr{H} .
\end{aligned}
$$

Thus $\left\{g_{j}\right\}_{j=1}^{\infty}$ is a Bessel sequence for $\mathscr{H}$. Now

$$
\begin{aligned}
& \left(\left.K^{*}\right|_{\overline{\mathrm{span}}\left\{f_{j}\right\}_{j=1}^{\infty}}\right)^{*} f=T U f \\
& =T \sum_{j=1}^{\infty}\left\langle U f, \delta_{j}\right\rangle \delta_{j}=\sum_{j=1}^{\infty}\left\langle U f, \delta_{j}\right\rangle T \delta_{j}=\sum_{j=1}^{\infty}\left\langle f, g_{j}\right\rangle f_{j} .
\end{aligned}
$$

(ii) $\Rightarrow$ (iii). $\quad$ For any $f \in \mathscr{H}$ and any $h \in$ $\overline{\operatorname{span}}\left\{f_{j}\right\}_{j=1}^{\infty}$, we see from (6) that

$$
\begin{aligned}
\left\langle\left(\left.K^{*}\right|_{\overline{\operatorname{span}}\left\{f_{j}\right\}_{j=1}^{\infty}}\right)^{*} f, h\right\rangle & =\sum_{j=1}^{\infty}\left\langle f, g_{j}\right\rangle\left\langle f_{j}, h\right\rangle \\
& =\left\langle f, \sum_{j=1}^{\infty}\left\langle h, f_{j}\right\rangle g_{j}\right\rangle .
\end{aligned}
$$

That is,

$$
\begin{aligned}
\left\langle f, K^{*} h\right\rangle & =\left\langle f,\left(\left.K^{*}\right|_{\overline{\operatorname{span}}\left\{f_{j}\right\}_{j=1}^{\infty}}\right) h\right\rangle \\
& =\left\langle f, \sum_{j=1}^{\infty}\left\langle h, f_{j}\right\rangle g_{j}\right\rangle,
\end{aligned}
$$

from which we conclude that $K^{*} h=\sum_{j=1}^{\infty}\left\langle h, f_{j}\right\rangle g_{j}$.

(iii) $\Rightarrow$ (i). Suppose that (7) holds. To prove that $\left\{f_{j}\right\}_{j=1}^{\infty}$ is a $K$-frame sequence, we only need to prove the lower bound inequality of the $K$-frame sequence. For any $g \in \overline{\operatorname{span}}\left\{f_{j}\right\}_{j=1}^{\infty}$ we have

$$
\begin{aligned}
\left\|K^{*} g\right\| & =\sup _{\|h\|=1}\left|\left\langle K^{*} g, h\right\rangle\right|=\sup _{\|h\|=1}\left|\sum_{j=1}^{\infty}\left\langle g, f_{j}\right\rangle\left\langle g_{j}, h\right\rangle\right| \\
& \leqslant\left(\sum_{j=1}^{\infty}\left|\left\langle g, f_{j}\right\rangle\right|^{2}\right)^{1 / 2} \sup _{\|h\|=1}\left(\sum_{j=1}^{\infty}\left|\left\langle h, g_{j}\right\rangle\right|^{2}\right)^{1 / 2} \\
& \leqslant \sqrt{D}\left(\sum_{j=1}^{\infty}\left|\left\langle h, g_{j}\right\rangle\right|^{2}\right)^{1 / 2}
\end{aligned}
$$

where $D$ is the Bessel bounds of $\left\{g_{j}\right\}_{j=1}^{\infty}$. Hence

$$
D^{-1}\left\|K^{*} g\right\|^{2} \leqslant \sum_{j=1}^{\infty}\left|\left\langle g, f_{j}\right\rangle\right|^{2}, \quad \forall g \in \overline{\operatorname{span}}\left\{f_{j}\right\}_{j=1}^{\infty} .
$$

Theorem $6 \mathrm{~A}$ sequence $\left\{f_{j}\right\}_{j=1}^{\infty} \subset \mathscr{H}$ is a $\mathrm{K}$ frame sequence if and only if there is $U \in$ $L\left(\overline{\operatorname{span}}\left\{f_{j}\right\}_{j=1}^{\infty}, \ell^{2}(\mathbb{N})\right)$ such that $U^{*} \delta_{j}=f_{j}$ for all $j \in$ $\mathbb{N}$ and $\left.R\left(\left(\left.K^{*}\right|_{\overline{\operatorname{span}}\left\{f_{j}\right.}\right\}_{j=1}^{\infty}\right){ }^{*}\right) \subset R\left(U^{*}\right)$, where $\left\{\delta_{j}\right\}_{j=1}^{\infty}$ is the canonical orthonormal basis for $\ell^{2}(\mathbb{N})$.

Proof: Assume first that $\left\{f_{j}\right\}_{j=1}^{\infty}$ is a $K$-frame sequence with bounds $C, D$ and the synthesis operator $T$. The definition gives

$$
C\left(\left.K^{*}\right|_{\overline{\operatorname{span}}\left\{f_{j}\right\}_{j=1}^{\infty}}\right)^{*}\left(\left.K^{*}\right|_{\overline{\operatorname{span}}\left\{f_{j}\right\}_{j=1}^{\infty}}\right) \leqslant T T^{*} .
$$

If we let $U=T^{*}$, we obtain $U^{*} \delta_{j}=T \delta_{j}=f_{j}$ for all $j \in \mathbb{N}$ and

$$
C\left(\left.K^{*}\right|_{\overline{\operatorname{span}}\left\{f_{j}\right\}_{j=1}^{\infty}}\right)^{*}\left(\left.K^{*}\right|_{\overline{\operatorname{span}}\left\{f_{j}\right\}_{j=1}^{\infty}}\right) \leqslant U^{*} U .
$$

From Lemma 2 it follows that

$$
R\left(\left(\left.K^{*}\right|_{\overline{\operatorname{span}}\left\{f_{j}\right\}_{j=1}^{\infty}}\right)^{*}\right) \subset R\left(U^{*}\right) .
$$

Conversely, since $U^{*} \delta_{j}=f_{j}$ for each $j \in \mathbb{N}$, we have

$$
\begin{aligned}
& \sum_{j=1}^{\infty}\left|\left\langle f, f_{j}\right\rangle\right|^{2}=\sum_{j=1}^{\infty}\left|\left\langle f, U^{*} \delta_{j}\right\rangle\right|^{2}=\sum_{j=1}^{\infty}\left|\left\langle U f, \delta_{j}\right\rangle\right|^{2} \\
& =\|U f\|^{2} \leqslant\|U\|^{2}\|f\|^{2}, \quad \forall f \in \overline{\operatorname{span}}\left\{f_{j}\right\}_{j=1}^{\infty} .
\end{aligned}
$$


Hence $\left\{f_{j}\right\}_{j=1}^{\infty}$ is a Bessel sequence for $\overline{\operatorname{span}}\left\{f_{j}\right\}_{j=1}^{\infty}$. Since $R\left(\left(\left.K^{*}\right|_{\overline{\operatorname{span}}\left\{f_{j}\right\}_{j=1}^{\infty}}\right)^{*}\right) \subset R\left(U^{*}\right)$, by Lemma 2 we know that there exists $\lambda>0$ such that $\left(\left.K^{*}\right|_{\overline{\operatorname{span}}\left\{f_{j}\right\}_{j=1}^{\infty}}\right)^{*}\left(\left.K^{*}\right|_{\overline{\operatorname{span}}\left\{f_{j}\right\}_{j=1}^{\infty}}\right) \leqslant \lambda U^{*} U$. Thus for each $f \in \overline{\operatorname{span}}\left\{f_{j}\right\}_{j=1}^{\infty}$,

$$
\begin{aligned}
\lambda^{-1}\left\|K^{*} f\right\|^{2} & =\lambda^{-1}\left\|\left.K^{*}\right|_{\overline{\operatorname{span}}\left\{f_{j}\right\}_{j=1}^{\infty}} f\right\|^{2} \\
& \leqslant\|U f\|^{2}=\sum_{j=1}^{\infty}\left|\left\langle f, f_{j}\right\rangle\right|^{2} .
\end{aligned}
$$

Hence $\left\{f_{j}\right\}_{j=1}^{\infty}$ is a $K$-frame sequence with bounds $\lambda^{-1},\|U\|^{2}$.

Corollary 1 A sequence $\left\{f_{j}\right\}_{j=1}^{\infty} \subset \mathscr{H}$ is a $K$-frame sequence for $\mathscr{H}$ if and only if the operator $T$ defined by (3) is well defined, linear bounded and $R\left(\left(\left.K^{*}\right|_{\overline{\operatorname{span}}\left\{f_{j}\right\}_{j=1}^{\infty}}\right)^{*}\right) \subset R(T)$.

Corollary 2 Let $\left\{f_{j}\right\}_{j=1}^{\infty} \subset \mathscr{H}$ be a Bessel sequence for $\overline{\operatorname{span}}\left\{f_{j}\right\}_{j=1}^{\infty}$. Then it is a $K$-frame sequence if and only if $R\left(\left(\left.K^{*}\right|_{\operatorname{span}\left\{f_{j}\right\}_{j=1}^{\infty}}\right)^{*}\right) \subset R(T)$.

\section{DUALS OF $K$-FRAMES IN HILBERT SPACES}

Definition 2 Let $\left\{f_{j}\right\}_{j=1}^{\infty}$ be a $K$-frame for $\mathscr{H}$. We call a Bessel sequence $\left\{g_{j}\right\}_{j=1}^{\infty}$ for $\mathscr{H}$ a dual $K$-frame of $\left\{f_{j}\right\}_{j=1}^{\infty}$ if

$$
K f=\sum_{j=1}^{\infty}\left\langle f, g_{j}\right\rangle f_{j}
$$

holds true for all $f \in \mathscr{H}$. In this case, we call $\left(\left\{f_{j}\right\}_{j=1}^{\infty},\left\{g_{j}\right\}_{j=1}^{\infty}\right)$ a dual $K$-frame pair.

Remark 2 If $K=\operatorname{Id}_{\mathscr{H}}$, then dual $K$-frames are just ordinary dual frames.

Remark 3 By (2), it is easily seen that if $\left\{g_{j}\right\}_{j=1}^{\infty}$ is a dual $K$-frame of $\left\{f_{j}\right\}_{j=1}^{\infty}$ then so is $\left\{\left(K^{\dagger} K\right)^{*} g_{j}\right\}_{j=1}^{\infty}$.

Remark 4 From Ref. 9 we know that every $K$-frame for $\mathscr{H}$ admits a dual $K$-frame.

It is well known that, in classical frame theory, the duals of a frame are necessarily frames. One may ask whether there is an analogue for $K$-frames. The answer is negative, as shown in the following example.

Example 3 Let $\left\{e_{j}\right\}_{j=1}^{\infty}$ be an orthonormal basis for $\mathscr{H}$ and define $K \in L(\mathscr{H})$ as follows:

$$
K e_{2 j}=e_{2 j}+e_{2 j-1}, \quad K e_{2 j-1}=0, j=1,2, \ldots .
$$

Then for each $f \in \mathscr{H}$ we have

$$
\begin{aligned}
K f & =K \sum_{j=1}^{\infty}\left\langle f, e_{j}\right\rangle e_{j} \\
& =K\left(\sum_{j=1}^{\infty}\left\langle f, e_{2 j}\right\rangle e_{2 j}+\sum_{j=1}^{\infty}\left\langle f, e_{2 j-1}\right\rangle e_{2 j-1}\right) \\
& =\sum_{j=1}^{\infty}\left\langle f, e_{2 j}\right\rangle\left(e_{2 j}+e_{2 j-1}\right) .
\end{aligned}
$$

It is easy to check that the adjoint operator $K^{*}: \mathscr{H} \rightarrow$ $\mathscr{H}$ is given by

$$
K^{*} f=\sum_{j=1}^{\infty}\left\langle f, e_{2 j}+e_{2 j-1}\right\rangle e_{2 j}, \quad \forall f \in \mathscr{H} .
$$

For $f \in \mathscr{H}$, since

$$
\begin{aligned}
\left\|K^{*} f\right\|^{2} & =\left\|\sum_{j=1}^{\infty}\left\langle f, e_{2 j}+e_{2 j-1}\right\rangle e_{2 j}\right\|^{2} \\
& =\sum_{j=1}^{\infty}\left|\left\langle f, e_{2 j}+e_{2 j-1}\right\rangle\right|^{2} \\
& \leqslant 2 \sum_{j=1}^{\infty}\left|\left\langle f, e_{2 j}\right\rangle\right|^{2}+2 \sum_{j=1}^{\infty}\left|\left\langle f, e_{2 j-1}\right\rangle\right|^{2} \\
& \leqslant 4\|f\|^{2},
\end{aligned}
$$

it follows that $\left\{f_{j}\right\}_{j=1}^{\infty}=\left\{e_{2 j}+e_{2 j-1}\right\}_{j=1}^{\infty}$ is a $K$ frame for $\mathscr{H}$. Clearly, $\left\{g_{j}\right\}_{j=1}^{\infty}=\left\{e_{2 j}\right\}_{j=1}^{\infty}$ is a Bessel sequence for $\mathscr{H}$. If there exists a constant $C>0$ such that $C\left\|K^{*} f\right\|^{2} \leqslant \sum_{j=1}^{\infty}\left|\left\langle f, g_{j}\right\rangle\right|^{2}$ for all $f \in \mathscr{H}$, then we have

$$
\begin{aligned}
\sum_{j=1}^{\infty}\left|\left\langle e_{1}, g_{j}\right\rangle\right|^{2} & =\sum_{j=1}^{\infty}\left|\left\langle e_{1}, e_{2 j}\right\rangle\right|^{2}=0 \\
& \geqslant C\left\|K^{*} e_{1}\right\|^{2}=C\left\|e_{2}\right\|^{2}=C,
\end{aligned}
$$

a contradiction. Thus $\left\{g_{j}\right\}_{j=1}^{\infty}$ is not a $K$-frame for $\mathscr{H}$.

Remark 5 One can check that a dual $K$-frame is necessarily a $K^{*}$-frame.

We now give a characterization of dual $K$ frames.

Theorem 7 Let $\left\{f_{j}\right\}_{j=1}^{\infty}$ be a $K$-frame for $\mathscr{H}$ with synthesis operator $T$, and $\left\{\delta_{j}\right\}_{j=1}^{\infty}$ be the canonical orthonormal basis for $\ell^{2}(\mathbb{N})$. The dual $K$-frames of $\left\{f_{j}\right\}_{j=1}^{\infty}$ are precisely the families $\left\{g_{j}\right\}_{j=1}^{\infty}=\left\{V \delta_{j}\right\}_{j=1}^{\infty}$, where $V: \ell^{2}(\mathbb{N}) \rightarrow \mathscr{H}$ is a linear bounded operator such that $K^{*}=V T^{*}$. 
Proof: For any $f \in \mathscr{H}$ we have

$$
\begin{aligned}
\sum_{j=1}^{\infty}\left|\left\langle f, g_{j}\right\rangle\right|^{2} & =\sum_{j=1}^{\infty}\left|\left\langle f, V \delta_{j}\right\rangle\right|^{2} \\
& =\left\|V^{*} f\right\|^{2} \leqslant\|V\|^{2}\|f\|^{2},
\end{aligned}
$$

showing that $\left\{g_{j}\right\}_{j=1}^{\infty}=\left\{V \delta_{j}\right\}_{j=1}^{\infty}$ is a Bessel sequence for $\mathscr{H}$. It is clear that $\left\{\left\langle f, f_{j}\right\rangle\right\}_{j=1}^{\infty}=$ $\sum_{j=1}^{\infty}\left\langle f, f_{j}\right\rangle \delta_{j}$ for all $f \in \mathscr{H}$. Thus

$$
\begin{aligned}
K^{*} f & =V T^{*} f=V\left\{\left\langle f, f_{j}\right\rangle\right\}_{j=1}^{\infty} \\
& =V \sum_{j=1}^{\infty}\left\langle f, f_{j}\right\rangle \delta_{j}=\sum_{j=1}^{\infty}\left\langle f, f_{j}\right\rangle g_{j} .
\end{aligned}
$$

Consequently, $K f=\sum_{j=1}^{\infty}\left\langle f, g_{j}\right\rangle f_{j}$, meaning that $\left\{g_{j}\right\}_{j=1}^{\infty}$ is a dual $K$-frame of $\left\{f_{j}\right\}_{j=1}^{\infty}$.

For the other implication, suppose that $\left\{g_{j}\right\}_{j=1}^{\infty}$ is a dual $K$-frame of $\left\{f_{j}\right\}_{j=1}^{\infty}$. Then the synthesis operator $U$ for $\left\{g_{j}\right\}_{j=1}^{\infty}$ satisfies the conditions. In fact, $\left\{g_{j}\right\}_{j=1}^{\infty}=\left\{U \delta_{j}\right\}_{j=1}^{\infty}$, and by the definition of a dual $K$-frame, $K^{*}=U T^{*}$.

Proposition 3.3 in Ref. 10 shows that a $K$-frame $\left\{f_{j}\right\}_{j=1}^{\infty}$ for $\mathscr{H}$ has a dual frame on the closed subspace $R(K)$ which is derived from a dual $K$ frame of $\left\{f_{j}\right\}_{j=1}^{\infty}$. It is natural to ask whether a $K$ frame admits a dual frame on the whole space $\mathscr{H}$. Unfortunately, the answer is negative.

Example 4 Let $\left\{e_{j}\right\}_{j=1}^{\infty}$ be an orthonormal basis for $\mathscr{H}$ and let $\left\{f_{j}\right\}_{j=1}^{\infty}$ be the same as in Example 2. Define a linear bounded operator as follows:

$$
K: \mathscr{H} \rightarrow \mathscr{H}, \quad K f=\sum_{j=1}^{\infty}\left\langle f, e_{2 j}\right\rangle e_{2 j}
$$

For any $f \in \mathscr{H}$ we compute that

$$
\begin{aligned}
&\left\|K^{*} f\right\|^{2}=\sum_{j=1}^{\infty}\left|\left\langle f, e_{2 j}\right\rangle\right|^{2} \leqslant \sum_{j=1}^{\infty}\left|\left\langle f, f_{j}\right\rangle\right|^{2} \\
&=\sum_{j=1}^{\infty}\left|\left\langle f, e_{2 j}\right\rangle\right|^{2}+\sum_{j=1}^{\infty} \frac{1}{(2 j-1)^{2}}\left|\left\langle f, e_{2 j-1}\right\rangle\right|^{2} \\
& \leqslant \sum_{j=1}^{\infty}\left|\left\langle f, e_{j}\right\rangle\right|^{2}=\|f\|^{2} .
\end{aligned}
$$

Hence $\left\{f_{j}\right\}_{j=1}^{\infty}$ is a $K$-frame for $\mathscr{H}$. Suppose that $\left\{f_{j}\right\}_{j=1}^{\infty}$ has a dual frame $\left\{g_{j}\right\}_{j=1}^{\infty}$. For any $k \in \mathbb{N}$, taking $e_{2 k-1} \in \mathscr{H}$, we have

$$
\begin{aligned}
e_{2 k-1} & =\sum_{j=1}^{\infty}\left\langle e_{2 k-1}, g_{j}\right\rangle f_{j}=\sum_{j=1}^{\infty}\left\langle e_{2 k-1}, f_{j}\right\rangle g_{j} \\
& =\sum_{j=1}^{\infty}\left\langle e_{2 k-1}, e_{2 j}\right\rangle g_{2 j}+\sum_{j=1}^{\infty}\left\langle e_{2 k-1}, \frac{e_{2 j-1}}{2 j-1}\right\rangle g_{2 j-1} .
\end{aligned}
$$

Thus $e_{2 k-1}=g_{2 k-1} /(2 k-1)$, and $g_{2 k-1}=(2 k-$ $1) e_{2 k-1}$ as a consequence. Now

$$
\begin{aligned}
& \sum_{j=1}^{\infty}\left|\left\langle e_{2 k-1}, g_{j}\right\rangle\right|^{2} \geqslant\left|\left\langle e_{2 k-1}, g_{2 k-1}\right\rangle\right|^{2} \\
&=(2 k-1)^{2} \rightarrow \infty \text { as } k \rightarrow \infty,
\end{aligned}
$$

which contradicts the fact that $\left\{g_{j}\right\}_{j=1}^{\infty}$ is a Bessel sequence for $\mathscr{H}$.

The converse of Proposition 3.3 in Ref. 10 still holds, provided an additional condition is added.

Theorem 8 Let $\left\{f_{j}\right\}_{j=1}^{\infty}$ be a Bessel sequence for $\mathscr{H}$ with frame operator $S$. If $\left\{f_{j}\right\}_{j=1}^{\infty}$ has a dual frame on $R(K)$ and $S(R(K)) \subset R(K)$, then it is a $K$-frame for $\mathscr{H}$.

Proof: Assume that $\left\{g_{j}\right\}_{j=1}^{\infty}$ is a dual frame of $\left\{f_{j}\right\}_{j=1}^{\infty}$ on $R(K)$. Each $f \in \mathscr{H}$ can be expressed as $f=d_{1}+$ $d_{2}$, where $d_{1} \in R(K)$ and $d_{2} \in(R(K))^{\perp}$. Then

$$
\begin{aligned}
\sum_{j=1}^{\infty}\left|\left\langle f, f_{j}\right\rangle\right|^{2}=\sum_{j=1}^{\infty} \mid\left\langle d_{1}\right. & \left.+d_{2}, f_{j}\right\rangle\left.\right|^{2} \\
=\sum_{j=1}^{\infty}\left|\left\langle d_{1}, f_{j}\right\rangle\right|^{2} & +\sum_{j=1}^{\infty}\left|\left\langle d_{2}, f_{j}\right\rangle\right|^{2} \\
& +2 \operatorname{Re} \sum_{j=1}^{\infty}\left\langle d_{1}, f_{j}\right\rangle\left\langle f_{j}, d_{2}\right\rangle .
\end{aligned}
$$

Noting $\sum_{j=1}^{\infty}\left\langle d_{1}, f_{j}\right\rangle f_{j}=S d_{1} \in S(R(K)) \subset R(K)$, we have $\sum_{j=1}^{\infty}\left\langle d_{1}, f_{j}\right\rangle\left\langle f_{j}, d_{2}\right\rangle=0$. Hence

$$
\sum_{j=1}^{\infty}\left|\left\langle f, f_{j}\right\rangle\right|^{2}=\sum_{j=1}^{\infty}\left|\left\langle d_{1}, f_{j}\right\rangle\right|^{2}+\sum_{j=1}^{\infty}\left|\left\langle d_{2}, f_{j}\right\rangle\right|^{2} .
$$

By Lemma 1, $\sum_{j=1}^{\infty}\left\langle d_{1}, f_{j}\right\rangle g_{j}$ converges and so does $\sum_{j=1}^{\infty}\left\langle d_{1}, f_{j}\right\rangle P_{R(K)} g_{j}$. Then for each $h \in R(K)$ we have

$$
\begin{aligned}
\left\langle h, d_{1}\right\rangle & =\sum_{j=1}^{\infty}\left\langle h, g_{j}\right\rangle\left\langle f_{j}, d_{1}\right\rangle=\left\langle h, \sum_{j=1}^{\infty}\left\langle d_{1}, f_{j}\right\rangle g_{j}\right\rangle \\
& =\left\langle h, \sum_{j=1}^{\infty}\left\langle d_{1}, f_{j}\right\rangle P_{R(K)} g_{j}\right\rangle .
\end{aligned}
$$


It follows that $d_{1}=\sum_{j=1}^{\infty}\left\langle d_{1}, f_{j}\right\rangle P_{R(K)} g_{j}$. Thus

$$
\begin{aligned}
\left\|K^{*} f\right\|^{4} & =\left\|K^{*}\left(d_{1}+d_{2}\right)\right\|^{4}=\left\|K^{*} d_{1}\right\|^{4} \\
& =\left\|\left\langle K^{*} d_{1}, K^{*} d_{1}\right\rangle\right\|^{2} \\
& =\left\|\sum_{j=1}^{\infty}\left\langle d_{1}, f_{j}\right\rangle\left\langle P_{R(K)} g_{j}, K K^{*} d_{1}\right\rangle\right\|^{2} \\
& \leqslant \sum_{j=1}^{\infty}\left|\left\langle d_{1}, f_{j}\right\rangle\right|^{2} \sum_{j=1}^{\infty}\left|\left\langle P_{R(K)} K K^{*} d_{1}, g_{j}\right\rangle\right|^{2} \\
& \leqslant D\|K\|^{2}\left\|K^{*} d_{1}\right\|^{2} \sum_{j=1}^{\infty}\left|\left\langle d_{1}, f_{j}\right\rangle\right|^{2} \\
& =D\|K\|^{2}\left\|K^{*} f\right\|^{2} \sum_{j=1}^{\infty}\left|\left\langle d_{1}, f_{j}\right\rangle\right|^{2},
\end{aligned}
$$

where $D$ is the Bessel bound of $\left\{g_{j}\right\}_{j=1}^{\infty}$. Hence

$$
\begin{aligned}
\sum_{j=1}^{\infty}\left|\left\langle f, f_{j}\right\rangle\right|^{2} & =\sum_{j=1}^{\infty}\left|\left\langle d_{1}, f_{j}\right\rangle\right|^{2}+\sum_{j=1}^{\infty}\left|\left\langle d_{2}, f_{j}\right\rangle\right|^{2} \\
\geqslant & \sum_{j=1}^{\infty}\left|\left\langle d_{1}, f_{j}\right\rangle\right|^{2} \geqslant D^{-1}\|K\|^{-2}\left\|K^{*} f\right\|^{2}
\end{aligned}
$$

There are two results on the perturbation of $K$ frames in a Hilbert space in Refs. 10,11. In the following we give a new perturbation result for $K$-frames where the associated dual $K$-frames are involved.

Theorem 9 Let $\left\{f_{j}\right\}_{j=1}^{\infty}$ be a $K$-frame for $\mathscr{H}$ with bounds $C, D$ and $\left\{g_{j}\right\}_{j=1}^{\infty}$ be a dual $K$-frame of $\left\{f_{j}\right\}_{j=1}^{\infty}$ with Bessel bound $D^{\prime}$. Assume that $\left\{h_{j}\right\}_{j=1}^{\infty}$ is a sequence in $\mathscr{H}$ which satisfies the following two conditions:

(i) $\lambda:=\sum_{j=1}^{\infty}\left\|h_{j}-f_{j}\right\|^{2}<\infty$;

(ii) $\mu:=\sum_{j=1}^{\infty}\left\|K^{\dagger}\right\|\left\|h_{j}-f_{j}\right\|\left\|g_{j}\right\|<1$.

Then $\left\{h_{j}\right\}_{j=1}^{\infty}$ is a $P_{L(R(K))} K$-frame for $\mathscr{H}$ with bounds $\left(D^{\prime}\right)^{-1}\left\|K^{\dagger}\right\|^{-2}\|K\|^{-2}(1-\mu)^{2},(\sqrt{\lambda}+\sqrt{D})^{2}$, where

$$
\begin{aligned}
& L: R(K) \rightarrow \mathscr{H}, \\
& \quad L f=\sum_{j=1}^{\infty}\left\langle f, P_{R(K)}\left(K^{\dagger}\right)^{*} g_{j}\right\rangle h_{j} .
\end{aligned}
$$

Proof: Define

$$
U: \ell^{2}(\mathbb{N}) \rightarrow \mathscr{H}, \quad U\left\{c_{j}\right\}_{j=1}^{\infty}=\sum_{j=1}^{\infty} c_{j} h_{j} .
$$

Then (i) implies that $U$ is well defined, linear, and bounded with $\|U\| \leqslant \sqrt{\lambda}+\sqrt{D}$. Thus

$$
\begin{aligned}
\sum_{j=1}^{\infty}\left|\left\langle f, h_{j}\right\rangle\right|^{2}=\left\|U^{*} f\right\|^{2} & \leqslant\|U\|^{2}\|f\|^{2} \\
& \leqslant(\sqrt{\lambda}+\sqrt{D})^{2}\|f\|^{2} .
\end{aligned}
$$

$L$ is well defined by Lemma 1 . Now, for any $f \in$ $R(K)$, we obtain

$$
\begin{gathered}
\|f-L f\|=\| \sum_{j=1}^{\infty}\left\langle K^{\dagger} f, g_{j}\right\rangle f_{j} \\
\quad-\sum_{j=1}^{\infty}\left\langle f, P_{R(K)}\left(K^{\dagger}\right)^{*} g_{j}\right\rangle h_{j} \| \\
=\| \sum_{j=1}^{\infty}\left\langle f, P_{R(K)}\left(K^{\dagger}\right)^{*} g_{j}\right\rangle f_{j} \\
\quad-\sum_{j=1}^{\infty}\left\langle f, P_{R(K)}\left(K^{\dagger}\right)^{*} g_{j}\right\rangle h_{j} \| \\
\leqslant \sum_{j=1}^{\infty}\left\|\left\langle f, P_{R(K)}\left(K^{\dagger}\right)^{*} g_{j}\right\rangle\left(f_{j}-h_{j}\right)\right\| \\
\leqslant \sum_{j=1}^{\infty}\left\|K^{\dagger}\right\|\left\|h_{j}-f_{j}\right\|\left\|g_{j}\right\|\|f\|=\mu\|f\| .
\end{gathered}
$$

Hence $(1-\mu)\|f\| \leqslant\|L f\|$ for all $f \in R(K)$. From this we conclude that the operator $L: R(K) \rightarrow L(R(K))$ is invertible with $\left\|L^{-1}\right\| \leqslant 1 /(1-\mu)$. It is trivial to show that $L(R(K))$ is closed. For any $h \in \mathscr{H}$ we have

$$
\begin{aligned}
P_{L(R(K))} K h & =L L^{-1} P_{L(R(K))} K h \\
& =\sum_{j=1}^{\infty}\left\langle L^{-1} P_{L(R(K))} K h, P_{R(K)}\left(K^{\dagger}\right)^{*} g_{j}\right\rangle h_{j} .
\end{aligned}
$$

For all $g \in \mathscr{H}$, again by Lemma 1, we obtain

$$
\begin{array}{r}
\left\langle P_{L(R(K))} K h, g\right\rangle=\left\langle\sum _ { j = 1 } ^ { \infty } \left\langle L^{-1} P_{L(R(K))} K h,\right.\right. \\
\left.\left.P_{R(K)}\left(K^{\dagger}\right)^{*} g_{j}\right\rangle h_{j}, g\right\rangle=\left\langle h, \sum_{j=1}^{\infty}\left\langle g, h_{j}\right\rangle \Delta g_{j}\right\rangle,
\end{array}
$$

where

$$
\Delta:=K^{*} P_{L(R(K))}\left(L^{-1}\right)^{*} P_{R(K)}\left(K^{\dagger}\right)^{*} .
$$

It follows that

$$
\left\langle h, K^{*} P_{L(R(K))} g-\sum_{j=1}^{\infty}\left\langle g, h_{j}\right\rangle \Delta g_{j}\right\rangle=0 .
$$


Hence

$$
K^{*} P_{L(R(K))} g=\sum_{j=1}^{\infty}\left\langle g, h_{j}\right\rangle \Delta g_{j} .
$$

For any $f \in \mathscr{H}$ we compute that

$$
\begin{aligned}
\left\|K^{*} P_{L(R(K))} f\right\| & =\sup _{\|x\|=1}\left|\sum_{j=1}^{\infty}\left\langle f, h_{j}\right\rangle\left\langle\Delta g_{j}, x\right\rangle\right| \\
\leqslant & \left(\sum_{j=1}^{\infty}\left|\left\langle f, h_{j}\right\rangle\right|^{2}\right)^{1 / 2} \\
& \times \sup _{\|x\|=1}\left(\sum_{j=1}^{\infty}\left|\left\langle\Delta^{*} x, g_{j}\right\rangle\right|^{2}\right)^{1 / 2} \\
\leqslant & \sqrt{D^{\prime}}\left\|K^{\dagger}\right\|\left\|L^{-1}\right\|\|K\| \\
& \times\left(\sum_{j=1}^{\infty}\left|\left\langle f, h_{j}\right\rangle\right|^{2}\right)^{1 / 2} \\
\leqslant & \frac{\sqrt{D^{\prime}}\left\|K^{\dagger}\right\|\|K\|}{1-\mu} \\
& \times\left(\sum_{j=1}^{\infty}\left|\left\langle f, h_{j}\right\rangle\right|^{2}\right)^{1 / 2} .
\end{aligned}
$$

Thus

$$
\begin{aligned}
& \left(D^{\prime}\right)^{-1}\left\|K^{\dagger}\right\|^{-2}\|K\|^{-2}(1-\mu)^{2}\left\|K^{*} P_{L(R(K))} f\right\|^{2} \\
& \quad \leqslant \sum_{j=1}^{\infty}\left|\left\langle f, h_{j}\right\rangle\right|^{2} .
\end{aligned}
$$

Acknowledgements: We thank the anonymous referees for valuable suggestions and comments which have led to a significant improvement of our manuscript. The research was partially supported by the Natural Science Foundation of Jiangxi, China (No. 20151BAB201007), the Science Foundation of Jiangxi Education Department (No. GJJ151061) and the National Natural Science Foundation of China (Nos. 11461057 and 11561057).

\section{REFERENCES}

1. Strohmer T, Heath RW (2003) Grassmannian frames with applications to coding and communication. Appl Comput Harmon Anal 14, 257-75.

2. Benedetto JJ, Powell A, Yilmaz O (2006) Sigma-delta $(\Sigma \Delta)$ quantization and finite frames. IEEE Trans Inform Theor 52, 1990-2005.

3. Sun WC (2010) Asymptotic properties of Gabor frame operators as sampling density tends to infinity. $J$ Funct Anal 258, 913-32.

4. Candès EJ, Donoho DL (2005) Continuous curvelet transform: II. Discretization and frames. Appl Comput Harmon Anal 19, 198-222.
5. Duffin RJ, Schaeffer AC (1952) A class of nonharmonic Fourier series. Trans Am Math Soc 72, 341-66.

6. Daubechies I, Grossmann A, Meyer Y (1986) Painless nonorthogonal expansions. J Math Phys 27, 1271-83.

7. Casazza PG (2000) The art of frame theory. Taiwan $J$ Math 4, 129-201.

8. Christensen O (2003) An Introduction to Frames and Riesz Bases, Birkhäuser, Boston.

9. Găvruţa L (2012) Frames for operators. Appl Comput Harmon Anal 32, 139-44.

10. Xiao XC, Zhu YC, Găvruţa L (2013) Some properties of $K$-frames in Hilbert spaces. Results Math 63, 1243-55.

11. Găvruţa L (2011) Perturbation of $K$-frames. Bul Ştiinţ Univ Politeh Timiş Ser Mat Fiz 56, 48-53.

12. Găvruţa L (2012) New results on frames for operators. An Univ Oradea Fasc Mat 19, 55-61.

13. Johnson PS, Ramu G (2015) Class of bounded operators associated with an atomic system. Tamkang $J$ Math 46, 85-90.

14. Douglas RG (1966) On majorization, factorization, and range inclusion of operators on Hilbert space. Proc Am Math Soc 17, 413-5. 